\title{
Nosocomial Candiduria in Critically Ill Patients Admitted to Intensive Care Units in Qazvin, Iran
}

\author{
Seyed Amir Ghiasian ${ }^{1,4, *}$; Mohammad Reza Aghamirian ${ }^{2}$; Golam Reza Eshghi ${ }^{3,4}$ \\ ${ }^{1}$ Department of Medical Parasitology and Mycology, School of Medicine, Hamadan University of Medical Sciences, Hamadan, IR Iran \\ ${ }^{2}$ Department of Medical Parasitology and Mycology, School of Medicine, Qazvin University of Medical Sciences, Qazvin, IR Iran \\ ${ }^{3}$ Department of Dermatology, School of Medicine, Hamadan University of Medical Sciences, Hamadan, IR Iran \\ ${ }_{4}^{4}$ Psoriasis Research Center, Hamadan University of Medical Sciences, Hamadan, IR Iran \\ *Corresponding author: Seyed Amir Ghiasian, Department of Medical Parasitology and Mycology, School of Medicine, Hamadan University of Medical Sciences, Hamadan, IR Iran. \\ Tel: +98-9188129953, E-mail: s.a.ghiasian@umsha.ac.ir
}

Received: June 10, 2014; Revised: June 20, 2014; Accepted: June 30, 2014

\begin{abstract}
Background: A broad variety of fungi, especially Candida species, are becoming increasingly common causes of urinary tract infections in hospital settings.

Objectives: The current cross-sectional descriptive study aimed to determine the causative agents, risk factors and incidence rate of candiduria in critically ill patients, hospitalized in intensive care units (ICUs) of four Iranian hospitals.

Patients and Methods: A total of 155 children and adult patients, admitted to ICUs of the four university educational hospitals, who demonstrated Candida-positive urine cultures, were examined. Samples were processed via mycological procedures by direct microscopy and culture.

Results: Candiduria was confirmed in 50 (32.26\%) patients and Candida albicans was the most frequently isolated species representing $60.0 \%$ of isolates, followed by $14.0 \%$ C.glabrata, $12.0 \%$ C. parapsilosis, $10.0 \%$ C. krusei, and $4.0 \%$ C. tropicalis. Most patients were female(58\%) with a mean age of 46.7 years old. Generally, $39.7 \%$ and $62 \%$ of adults and children showed candiduria, respectively. The commonest predisposing factors were antibiotic therapy (98.0\%), urinary catheterization (92.0\%), corticotherapy (84.0\%), being female (42.6\%), use of feeding tube (56\%), and extended hospitalization, respectively.

Conclusions: The high frequency of candiduria in ICU patients can be decreased by shortening the duration of urinary catheterization, avoiding extra antibiotics and corticosteroids, as well as controlling the predisposing factors and underlying conditions.
\end{abstract}

Keywords: Candidiasis; Yeasts; Intensive Care Unit; Candida albicans

\section{Background}

Fungi are one of the common pathogens causing nosocomial infections. Among hospital wards, intensive care unit (ICU) has the highest incidence of nosocomial infections (1). Numerous patients are hospitalized every day, some of whom require urinary catheterization. Urinary tract infections (UTIs) are serious and common complications in hospitalized critically ill patients, representing about $30-40 \%$ of nosocomial infections (2). Urinary catheter has been one of the most important UTI causing factors $(1,3)$. The longer the catheter remains, the higher content of infectious organisms appear in urine (1). Hauer et al. upon conducting a study of hospital acquired infections in hospital ICUs in Germany introduced pneumonia as the most common hospital acquired infection, with UTI and septicemia coming in the second and third places, respectively (4). In a study on prevalence of infections in European ICUs, UTI was the third most common infection (18\%) in hospitalized patients (5). Long ICU stay, age over 65 , being a female and antibiotic consumption have been effective factors in acquiring candiduria (6). Furthermore, fungal UTIs of hospitalized patients, especially with Candida spp., have increased during the past decade. However, there are no definite criteria to differentiate colonization from UTI caused by Candida spp.(1). The practices of American physicians upon positive urinary cultures for Candida spp. have been investigated. they started antifungal treatment based on positive urinary cultures in most of the cases (60.2\%); the treatment was based on a single positive culture without any document to confirm the infection or risk for Candida invasion (7). According to a study on epidemiology of UTIs, increase of these infections resulted in longer hospital stay and increased hospital charges (8).

In Iran, ability of Candida species in biofilm formation on urinary catheters of ICU patients has been assessed (9). In another study, antifungal susceptibilities of 93 species of yeasts and yeast-like organisms isolated from urine samples of patients with candiduria were evaluated (10). Furthermore, the incidence of candiduria in critically ill children and adults has been considered by some researchers (10-12).

\section{Objectives}

This comprehensive multicenter and clinico-epide-

Copyright (C 2014, Hamadan University of Medical Sciences; Published by Safnek. This is an open-access article distributed under the terms of the Creative Commons Attribution License, which permits unrestricted use, distribution, and reproduction in any medium, provided the original work is properly cited. 
miological study aimed to determine the incidence, predisposing factors, and microbiological characteristics of etiological agents involved in nosocomial candiduria, associated with indwelling urinary catheters in critically ill adults and children admitted to four different ICUs in Qazvin, Iran.

\section{Patients and Methods}

This descriptive analytical study was run for three months between November 2008 to January 2009 on 155 adults and children, consecutively admitted to the ICUs of four educational hospitals, including General, Women's, Surgery, and Children's hospitals of Qazvin University of Medical Sciences and Health Services (the contributing centers are listed under the acknowledgements section). For all the enrolled patients, information including demographic features (age and gender), presence of risk factors (catheterization and treatment with antibiotics, corticosteroids, and antifungal drugs), underlying diseases (diabetes, anemia and feeding tube infection) as well as concomitant infections was gathered through questionnaires.

All the patients who underwent urethral catheterization, had negative urine cultures at the time of admission, showed positive yeast urine cultures 72 hours after the insertion of urinary catheter, and had fever above $38^{\circ} \mathrm{C}$ and pyuria were investigated in this study. The exclusion criteria were patients who were immune-suppressed and neutropenic, had fungal infections at the hospital admission, catheterized patients with positive fungal urine cultures at the admission time, less than 24 hours of hospitalization, and those with indwelling urinary catheters with undefined dates of insertion. Out of the four surveyed hospitals, one was general medical and the rest were specialized, including obstetrics and gynecology, surgical and emergency, and children's hospitals.

In all patients, the initial urine sample was obtained on admission to the ICU, three days after the hospitalization, and routine urine cultures were carried out once a week thereafter. The extra samples were obtained upon discretion of the attending physician, if needed. These evaluations were continued daily until the patient's catheter was detached, she/he was discharged from the ICU, or until the patient died.

In this study, the urine samples were mainly obtained from catheters, and in $22.2 \%$ of children, it was taken through suprapubic aspiration. The urine specimens were examined promptly by direct microscopy and culture. Direct microscopic examinations of urine specimens were carried out by KOH and Gram/Geimsa staining. Presence of yeast cells with budding cells and pseudohyphae were identifying features of positive smears. The yeast fungi, observed on direct microscopy of urine samples, were quantitated by streaking of a loopful of undiluted urine across a Sabouraud dextrose agar (BioMerieux, Marcy-1, Etoile, France) with chlorampheni- col (SC) culture medium. The degree of growth was also estimated after overnight incubation at $37^{\circ} \mathrm{C}$ and counting the number of fungal colonies. Candiduria was defined as presence of $\geq 10^{5}$ colony forming units (cfu) of Candida spp. per milliliter of urine specimen, collected at least 72 hours after hospital admission.

Candida species were differentiated based on chlamydospore production on corn meal agar with Tween 80 (Hardy Diagnostics, Santa Maria, California, USA) and on chlamydospore agar (Difco). They were identified using colony color on CHROMagar Candida medium (Becton Dickinson, Sparks, MD, USA) and confirmed by API 20C AUX identification kit system (Byomerieux, Lyon, France) (1, 6).

\subsection{Statistical Analysis}

Statistical analyses were performed using SPSS version 9.0 (SPSS Inc., Chicago, Illinois). Quantitative variables were expressed as mean and standard deviation (SD) and qualitative variables as the percentage of distribution for each category. Categorical variables were compared using the chi-square or Fisher's exact tests. The t-test or the Mann-Whitney U test was used for comparison of normally and non-normally distributed variables, respectively. The results were considered significant if $\mathrm{P}<0.05$.

\section{Results}

A total of 155 hospitalized patients met the inclusion criteria. Out of 50 patients with UTI, 58\% were female with a mean age of 46.7 years old. The age ranged from three months to 81 years old, with a mean of 54.5 (Table 1). Among 155 hospitalized patients, 29 (18.7\%) were children, of which $62 \%$ had Candida-positive urine cultures. Patients who had positive urine cultures (at least $10^{5} \mathrm{CFU} /$ $\mathrm{mL}$ ) for Candida species three days after the insertion of indwelling urinary catheters were considered as the ones with candiduria and the risk factors were analyzed in these patients. The urine samples of adults were obtained from catheters, and in children, they were taken through suprapubic aspiration.

Candiduria was recovered in 50 (32.26\%) patients, and in one or more urine samples, five different Candida species were isolated during the ICU stay. Patients with candiduria had a mean \pm SD age of $47.2 \pm 13.2$ years old. The frequency was higher among women (58\%) with a mean age of 46.7 years old. Candida albicans was the most frequently isolated species representing $60.0 \%$ of isolates, followed by $7(14.0 \%)$ C. glabrata, 6 (12.0\%) C. parapsilosis, $5(10.0 \%)$ C. krusei, and $2(4.0 \%)$ C. tropicalis. The commonest predisposing factors, significantly associated with recovery of Candida spp. in urine, were antibiotic therapy (98.0\%), urethral catheterization (92.0\%), corticotherapy ( $84.0 \%)$, being female ( $42.6 \%$ ), use of feeding tube $(56 \%)$ and extended hospitalization, respectively. The commonest concurrent exposures in patients with candiduria were antibiotic therapy and urethral catheterization.

In this study, only $6(10 \%)$ out of 50 candiduria cases 
were associated with diabetes mellitus. Different nonalbicans Candida. (excluding C. albicans) in one urinary sample were only isolated in four cases. In two cases, the initial strains of C. albicans were substituted by C. tropicalis in consequent weeks, and in two other cases, coexistence of C. albicans and C. krusei was observed. In 3 (6\%) cases, isolation of Candida species in urine cultures was accompanied by isolation of Escherichia coli.

Moreover, a significant difference $(\mathrm{P}=0.001)$ was observed between candiduria, female sex, and corticosteroids consumption; but, no significant difference was found between candiduria and diabetes mellitus. Candiduria was frequently associated with antibiotic usage $(\mathrm{P}=$ $0.002)$ and urinary catheter $(\mathrm{P}=0.025)$. Moreover, regarding the use of feeding tube, a significant difference was observed between patients with and without candiduria admitted to the ICUs ( $56 \%$ vs. $37 \%, \mathrm{P}=0.027$ ) (Table 1).

The underlying diseases were classified as medical, surgical, and trauma in $48 \%, 20 \%$, and $20 \%$ of the patients, respectively. The average length of ICU stay was 11 days with a standard deviation of seven days. Patients with candiduria compared with those without candiduria showed no significantly higher ICU mortality rate (34\% vs. $27.6 \%$, $\mathrm{P}=0.252$ ). Anemia, also as an underlying condition, was present in $41.9 \%$ of patients and there was no significant difference between patients with and without funguria.

\section{Discussion}

This was the first large multicenter clinico-epidemiological study focusing on 155 patients hospitalized in ICUs of Qazvin province educational hospitals, who inflicted with candiduria. Several reports indicated that nosocomial UTIs frequently encountered in patients admitted to ICUs $(1,6,8,9,11,12)$. The incidence of candiduria has significantly increased among ICU patients, especially in those with indwelling urinary catheters (1). Candiduria was seen in $32.26 \%$ of ICU patients in this study. Reports from several countries indicate that candiduria is an increasing imperative problem among patients admitted to ICUs and may lead to invasive candidiasis, especially in those with critical conditions $(6,8,13-15)$.

The high incidence of funguria in Iranian ICU patients should be considered as very critical (9-12). According to

Table 1. Information on Demographics, Underlying Conditions and Risk Factors Associated With Critically Ill Patients With Candiduria ${ }^{a, b}$

\begin{tabular}{|c|c|c|c|}
\hline \multirow[t]{2}{*}{ Variable } & \multicolumn{3}{|c|}{ Candida Spp. in Urine } \\
\hline & Positive $($ No. $=50)$ & Negative $($ No. $=105)$ & P value \\
\hline \multicolumn{4}{|l|}{ Sex } \\
\hline Male & $21(24.1 \%)$ & $66(75.9 \%)$ & $<0.001$ \\
\hline Female & $29(42.6 \%)$ & $39(57.4 \%)$ & $<0.015$ \\
\hline Age, $y$, mean \pm SD & $47.2 \pm 13.2$ & $52 \pm 11.6$ & $<0.656$ \\
\hline Number of adults & $32(25.4 \%)$ & $94(74.6 \%)$ & $<0.001$ \\
\hline Number of children & $18(62 \%)$ & $11(37.9 \%)$ & $<0.001$ \\
\hline ICU stay, d, mean \pm SD & $11 \pm 7$ & $23.1 \pm 9.6$ & $<0.001$ \\
\hline \multicolumn{4}{|l|}{ Diagnosis at the time of ICU admission ${ }^{b}$} \\
\hline Medical diagnosis & $24(48 \%)$ & $47(44.8 \%)$ & $<0.158$ \\
\hline Surgical diagnosis & $10(20 \%)$ & $25(23.8 \%)$ & $<0.456$ \\
\hline Trauma & $10(20 \%)$ & $20(19 \%)$ & $<0.510$ \\
\hline Ischemic heart disease & $6(12 \%)$ & $9(8.6 \%)$ & $<0.536$ \\
\hline Hematological diagnosis & $2(4 \%)$ & $4(3.8 \%)$ & $<0.931$ \\
\hline ICU mortality rate & $17(34 \%)$ & $29(27.6 \%)$ & $<0.252$ \\
\hline \multicolumn{4}{|l|}{ Risk factors and underlying conditions } \\
\hline Antibiotic consumption & $49(98 \%)$ & $83(79 \%)$ & $<0.002$ \\
\hline Corticosteroid consumption & $42(84 \%)$ & $51(48.6 \%)$ & $<0.000$ \\
\hline Urinary catheterization & $46(92 \%)$ & $81(77.1 \%)$ & $<0.025$ \\
\hline Antifungal consumption & $2(4 \%)$ & $3(2.9 \%)$ & 0.704 \\
\hline Anemia & $23(46 \%)$ & $42(40 \%)$ & 0.225 \\
\hline Feeding tube usage & $28(56 \%)$ & $39(37 \%)$ & $<0.027$ \\
\hline Insulin-dependent diabetes mellitus & $5(10 \%)$ & $20(19.1 \%)$ & 0.628 \\
\hline
\end{tabular}

\footnotetext{
a Abbreviation: ICU, intensive care unit.

b Some patients were in more than one category
} 
Nassoura et al. (1993) presence of Candiduria in high risk patients might be indicative of UTI or systemic infection (16). UTIs are common problems of critical diseases which lead to increased patients' morbidity, but not mortality (8). There is still a controversy over the mortal effect of candiduria in critically ill patients. Although a large body of literature suggests that ICU-acquired UTIs are associated with increased patients' morbidity rates, they generally do not appear to individually increase the risk for death. In this study among the four surveyed ICUs in Iran, patients with candiduria compared with those without candiduria did not show significantly higher mortality rates ( 34 vs. $27.6 \%, \mathrm{P}=0.252$ ). These results were comparable with that reported by Bagshaw and Laupland (8), but in contrast with a study carried out by the EPCAN study group, in which significant differences were found in ICU mortality rates in 70 surveyed Spanish hospitals (6). Another study in Argentina also showed an increased risk for death related to increase of UTI in patients admitted to ICUs (13).

From an etiological standpoint, the most frequent fungal pathogens encountered have been $C$. albicans and non-albicans Candida species (8). Currently, 10-15\% of UTIs are caused by Candida species $(1,6)$. A great majority of fungal UTIs are caused by Candida species and the most frequent organism is C. albicans, followed by C. glabrata, C. tropicalis, and C. krusei $(12,16,17)$. During the last decades, the worldwide increase in the incidence of nonalbicans Candida spp. with highly variable susceptibility to fluconazole has been of special concern among critically ill patients. Specific identification of Candida spp. in patients with candiduria provides important help in treatment selection, since C. glabrata, C. krusei, C. tropicalis, and C. parapsilosis may be less sensitive and naturally resistant to certain antifungal drugs like fluconazole (18, 19). Furthermore, the antifungal susceptibility test on some Candida species isolated from Iranian patients with candiduria has revealed significant resistance against ketoconazole and fluconazole (10). In the present study, Candida spp. were the most implicated agents and C. albicans outnumbered all other species, accounting for $60.0 \%$. Predominance of C. albicans in our finding was in conformity with some other reports $(6,8,12-15,17,20,21)$. In a large multicenter surveillance study carried out by Kauffman et al. C. albicans was the commonest etiological agent (51.8\%) of funguria in the United States (22). The prevalence of non-albicans Candida species as the etiological causes of fungal UTI has significantly increased over the past decade $(1,6)$. In our study, non-albicans Candida was recovered in 20 (40\%) cases and C. glabrata was the most common species isolated after C. albicans. Furthermore, isolation of C. glabrata as the second most frequently recovered yeast in our patients with candiduria was comparable with what Alvarez et al. found in a large majority of surveyed Spanish hospitals (6). Our findings were also in concordance with an Iranian study on 744 urine samples (12) and a Brazilian study conducted on urine samples from 153 ICU patients (15). In fact, reports have indicated that the capacity of C. glabrata isolates for biofilm formation may be a potential virulence factor responsible for catheter-related candidemia in ICU patients $(15,23)$.

Due to the capacity of C. parapsilosis family for biofilm formation, they have emerged as main opportunistic and nosocomial pathogens $(23,24)$. This may relate to the ability to adhere to skin, plastic, and multiply insolutions containing glucose. Interestingly, $60 \%$ of the C. parapsilosis isolated in our study were obtained from patients with diabetes mellitus $(23,24)$. In addition, it is not all members of the C. parapsilosis family can be virulent, and between them, C. metapsilosis is an environmental organism, rarely isolated from clinical samples (23). In spite of the importance of $C$. parapsilosis differentiation in clinical studies carried out on UTIs, it was not investigated in the current survey. C. tropicalis, similar to some other studies $(6,14,15)$, had the lowest frequency. Furthermore, in two candiduria cases, initial strains of $C$. albicans were substituted by C. tropicalis in consequent weeks, possibly due to its antifungal resistance.

Antibiotic therapy is one of the most important risk factors contributed to nosocomial Candida spp. colonizations/infections by suppressing endogenous bacterial flora and decrease of nonbacterial emerging flora, mainly in colorectal tract and in areas close to the ureteral meatus $(1,6)$. In the current study, antibiotic therapy was the principal (98.0\%) predisposing factor and a total of 48 patients (out of 50) used broad-spectrum antibiotics. In support of our study, other researchers from different countries especially Iran concluded that broad-spectrum antibiotic therapy can be one of the most important predisposing factors for development of candiduria in patients admitted to ICUs $(1,6,8,12,25,26)$. Nonetheless, some other studies found greater prevalence of this factor and showed that all critically ill patients with nosocomial candiduria had received antibiotics $(15,27)$.

In recent years, presence of UTIs in patients admitted to ICUs and requiring prolonged urinary catheterization has increased and Candida spp. have been the causative agents of $10-15 \%$ of these infections $(1,6,26)$. Nonetheless, a large body of literature suggests that indwelling urinary catheter has been one of the most imperative risk factor contributing to the overgrowth of Candida spp. and development of candiduria in ICU patients $(1,6,22$, $27,28)$. In our study, urinary catheterization was the second common predisposing factor (92.0\%) among Iranian patients admitted to ICUs. This was comparable with the results of Passos et al. who also reported using urinary catheters in $92.6 \%$ of ICU patients with candiduria (15). This observation was also documented by Richards et al. indicating that more than $95 \%$ of all UTIs occurred in the USA were related to indwelling urinary catheters (28). In accordance with our study, most studies identified that extended ICU stay may increase the risk for ICU-acquired UTIs $(6,8,13,26)$. In contrast, the recently achieved results 
in educational hospitals of Ahvaz, Iran, show that only $18.7 \%$ of hospitalized patients were fully catheterized (12). The increased incidence of nosocomial candiduria has also been associated with corticosteroid treatment (17, 18). In spite of the importance of treatment with corticosteroids in patients with ICU-acquired UTIs, to which most of the studies referred $(17,18)$, there is a little information about its relationship with candiduria. Irrespective of antibiotic therapy, in our study, corticotherapy was the third most common risk factor (84\%) contributed to UTIs. Similar results were found by Guler et al. showing that the risk for candiduria increased by 1.4 folds in association with corticosteroid administration (21).

The genitourinary system is a main source of Candida spp. and the major group of patients with UTIs is adult females $(17,18)$. In our study, prevalence of candiduria in females and males was 42.6 and $24.1 \%$, respectively. According to Delgado et al. frequency of candiduria was higher among females (6.3\% vs 1.7\%, P = 0.001) (25). Passos et al. (15), Kobayashi et al. (27) and Alvarez et al (6) also reported that respectively, $61.6 \%, 57.8 \%$, and $49.1 \%$ of surveyed patients were females. Some reasons for higher prevalence of candiduria in females compared with males may be their shorter urethral length, transmission from the genital tract to the urinary tract, and the antiCandida activity of prostatic fluid in males. In a study on eight patients with candiduria, the yeast species isolated from five urine and vaginal secretions were the same (14). The placement of feeding tube or enteral feeding in our patients was temporary or lifelong, according to their acute conditions or chronic disabilities. In addition to using urinary catheters, $56 \%$ of our patients used feeding tubes and of interest, the obtained results showed that there was a significant difference $(P=0.027)$ between candiduria and using feeding tubes in patients admitted to Iranian ICUs. Although most studies have focused on the effects of parenteral feeding on critically ill patients, the effects of feeding tube on development of ICU-acquired UTI has not been well studied yet. Use of indwelling devices such as catheters, feeding tubes and other plastic surgical devices has been recognized as one of the important factors associated with candidiasis, due to biofilm formation (20).

Diabetic patients are at increased threat of candiduria by predisposition to Candida colonizations/infections via increase of fungal growth in the urinary tract and by impairment of phagocytic activity of macrophages as a result of lowered host resistance and fungal invasion (1, $17,18)$. Although few studies have investigated diabetes mellitus as a risk factor for candiduria, in this study, 10\% of patients with UTI had diabetes mellitus. Similar results were found by Zarei-Mahmoudabadi et al. who reported this rate as $11.4 \%$ in their investigation (12). Bukhary upon conducting a literature review on candiduria management during a 37-year period, stated that the risk to develop candiduria was increased by two folds in the presence of diabetes mellitus (30). Therefore, control of this risk factor can be one of the most common approaches to manage candiduria in diabetic patients (1).

Among the underlying conditions associated with UTIs, anemia has not been extensively evaluated to date. A study in Brazil addressed anemia as an underlying condition in $22(41.2 \%)$ patients with funguria (15). The prevalence of anemia as an underlying condition was $41.9 \%$ in our patients and although patients with candiduria compared with those without candiduria showed a higher anemia rate (34\% vs. $27.6 \%$ ), this difference was not significant $(\mathrm{P}=0.252)$. The incidence of Candida UTIs in hospitalized pediatric patients has been increased during the recent years (31). In the present study, the frequency of candiduria was higher among children than adults ( $62 \%$ vs $25.4 \%, \mathrm{P}=0.001$ ) (Table 1). Bryant et al. (1999) (32) upon conducting a 12-year study in the large tertiary care neonatal ICUs in the USA, established the overall incidence of candiduria to be $0.5 \%$. In contrast, another study in the USA (33) demonstrated a greater prevalence of Candida species causing UTI in $42 \%$ of patients. In the current study, some of the major reasons that contributed to the high positive candiduria rate of children may be prolonged usage of urinary drainage catheters and long-term/inappropriate antibiotic or corticosteroid treatment. In spite of the aforementioned risk factors, over $66 \%$ of the children who showed candiduria had a history of preterm birth/very low birth weight (< $1500 \mathrm{~g}$ ) as well as severe or frequent infections.

Different results yielded from studies on infections caused by urinary catheter and candiduria have led to a false presumption that the majority of yeasts found in urine cultures of such patients are of no importance, whereas presence of yeasts in urine can cause serious problems for these patients. Many of the yeasts appear in urine following urinary catheterization can cause problems for the patient according to the patient's conditions and the length of catheter dwelling time, if not controlled (34).

In conclusion, the results showed that nosocomial candiduria by Candida species had a high prevalence among children and adults in different hospital ICUs in Qazvin. The main predisposing factors were antibiotic and corticotherapy, urinary catheterization, and extended hospitalization, respectively. The incidence of nosocomial UTIs can be decreased in ICU patients by shortening the duration of urinary catheterization, avoiding extra antibiotics and corticosteroids prescription, and finally, controlling the predisposing factors and underlying conditions.

\section{Acknowledgements}

The authors are grateful of physicians and nurses of the Abuali General Hospital, Kowsar Gynecology Hospital, Shahid Radjaei Surgery Hospital, and Ghods Children's Hospital for their substantial helps in this study.

\section{References}

1. Weinstein, Lundstrom T, Sobel J. Nosocomial candiduria: a review. Clin Infect Dis. 2001;32(11):1602-7. 
2. Eriksen HM, Iversen BG, Aavitsland P. Prevalence of nosocomial infections in hospitals in Norway, 2002 and 2003. J Hosp Infect. 2005;60(1):40-5.

3. Gaynes RP, Culver DH, Horan TC, Emori TG, Horan TC, Banerjee SN, Edwards JR, et al. The National Nosocomial Infections Surveillance System: plans for the 1990s and beyond. Am J Med. 1991;91(3B):116S-20S.

4. Hauer T, Lacour M, Gastmeier P, Schulgen G, Schumacher M, Ruden $\mathrm{H}$, et al. Nosocomial infections intensive care units. A nation-wide prevalence study. Anaesthesist. 1996;45(12):1184-91.

5. Vincent JL, Bihari DJ, Suter PM, Bruining HA, White J, NicolasChanoin $\mathrm{MH}$, et al. The prevalence of nosocomial infection in intensive care units in Europe. Results of the European Prevalence of Infection in Intensive Care (EPIC) Study. EPIC International Advisory Committee. JAMA. 1995;274(8):639-44.

6. Alvarez-Lerma F, Nolla-Salas J, Leon C, Palomar M, Jorda R, Carras co N, et al. Candiduria in critically ill patients admitted to intensive care medical units. Intensive Care Med.2003;29(7):1069-76.

7. Ayeni O, Riederer KM, Wilson FM, Khatib R. Clinicians' reaction to positive urine culture for Candida organisms. Mycoses. 1999;42(4):285-9.

8. Bagshaw SM, Laupland KB. Epidemiology of intensive care unit-acquired urinary tract infections. Curr Opin Infect Dis. 2006;19(1):67-71.

9. Mohammadi P, Shoaie N, Roudbar M. . Isolation and Detection of Yeast Biofilms From Urine Catheters of Infectious Patients. jjm microbiol. 2012;5(4):533-6.

10. Zarei MA, Zarrin M, Beheshti F. Antifungal susceptibility of Candida species isolated from. JJ Microbiol. 2012;5(4):533-6.

11. Seifi Z, Azish M, Salehi Z, Zarei Mahmoudabadi A, Shamsizadeh A. Candiduria in children and susceptibility patterns of recovered Candida species to antifungal drugs in Ahvaz. J Nephropathol. 2013;2(2):122-8.

12. Zarei-Mahmoudabadi A, Zarrin M, Ghanatir F, Vazirianzadeh B. Candiduria in hospitalized patients in teaching hospitals of Ahvaz. Iran J Microbiol. 2012;4(4):198-203.

13. Rosenthal VD, Guzman S, Orellano PW. Nosocomial infections in medical-surgical intensive care units in Argentina: attributable mortality and length of stay. Am J Infect Control. 2003;31(5):291-5.

14. Febre N, Silva V, Medeiros EA, Wey SB, Colombo AL, Fischman O. Microbiological characteristics of yeasts isolated from urinary tracts of intensive care unit patients undergoing urinary catheterization. J Clin Microbiol.1999;37(5):1584-6.

15. Passos XS, Sales WS, Maciel PJ, Costa CR, Miranda KC, Lemos Jde A, et al. Candida colonization in intensive care unit patients' urine. Mem Inst Oswaldo Cruz. 2005;100(8):925-8.

16. Nassoura Z, Ivatury RR, Simon RJ, Jabbour N, Stahl WM. Candiduria as an early marker of disseminated infection in critically ill surgical patients: the role of fluconazole therapy. J Trauma.
1993;35(2):290-4

17. Krcmery S, Dubrava M, Krcmery V, Jr. Fungal urinary tract infections in patients at risk. Int J Antimicrob Agents. 1999; 11(34):289-91.

18. Lundstrom T, Sobel J. Nosocomial candiduria: a review. Clin Infect Dis. 2001;32(11):1602-7.

19. Rex JH, Rinaldi MG, Pfaller MA. Resistance of Candida species to fluconazole. Antimicrob Agents Chemother.1995;39(1):1-8

20. Odds FC. Ecology and epidemiology of Candida species. Zenrrulbl Bakreriol Microbiol Hyg. 1984;257(2):207-12.

21. Guler S, Ural O, Findik D, Arslan U. Risk factors for nosocomial candiduria. Saudi Med J.2006;27(11):1706-10.

22. Kauffman CA, Vazquez JA, Sobel JD, Gallis HA, McKinsey DS, Karchmer AW, et al. Prospective multicenter surveillance study of funguria in hospitalized patients. The National Institute for Allergy and Infectious Diseases (NIAID) Mycoses Study Group. Clin Infect Dis. 2000;30(1):14-8.

23. Trofa D, Gacser A, Nosanchuk JD. Candida parapsilosis, an emerging fungal pathogen. Clin Microbiol Rev. 2008;21(4):606-25.

24. van Asbeck EC, Huang YC, Markham AN, Clemons KV, Stevens DA Candida parapsilosis fungemia in neonates: genotyping results suggest healthcare workers hands as source, and review of published studies. Mycopathologia.2007;164(6):287-93.

25. Delgado J, Calvo N, Gomis A, Perez-Flores I, Rodriguez A, Ridao $\mathrm{N}$, et al. Candiduria in renal transplant recipients: incidence, clinical repercussion, and treatment indication. Transplant Proc. 2010;42(8):2944-6.

26. Chabasse D. [Yeast count in urine. Review of the literature and preliminary results of a multicenter prospective study carried out in 15 hospital centers]. Ann Fr Anesth Reanim. 2001;20(4):400-6.

27. Kobayashi CC, de Fernandes OF, Miranda KC, de Sousa ED, Silva Mdo R. Candiduria in hospital patients: a study prospective. Mycopathologia. 2004;158(1):49-52.

28. Richards MJ, Edwards JR, Culver DH, Gaynes RP. Nosocomial in fections in combined medical-surgical intensive care units in the United States. Infect Control Hosp Epidemiol. 200 0;21(8):510-5.

29. Odds FC. Ecology and epidemiology of Candida species. Zentralb Bakteriol Mikrobiol Hyg A. 1984;257(2):207-12.

30. Bukhary ZA. Candiduria: a review of clinical significance and management. Saudi J Kidney Dis Transpl. 2008;19(3):350-60.

31. Chang SL, Shortliffe LD. Pediatric urinary tract infections. Pediatr Clin North Am. 2006;53(3):379-400.

32. Bryant K, Maxfield C, Rabalais G. Renal candidiasis in neonates with candiduria. Pediatr Infect Dis J. 1999;18(11):959-63.

33. Phillips JR, Karlowicz MG. Prevalence of Candida species in hospital-acquired urinary tract infections in a neonatal intensive care unit. Pediatr Infect Dis J.1997;16(2):190-4.

34. Traboulsi R, Kanafani ZA, Kanj SS. Fungal infections of the genitourinary tract. J Med Liban. 2004;52(4):202-9. 